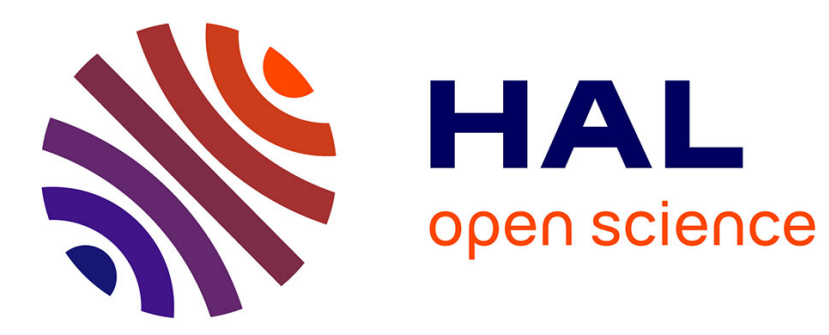

\title{
Subcritical transition to turbulence in a sudden circular pipe expansion
}

Benoît Lebon, Jorge Peixinho, Shun Ishizaka, Yuji Tasaka

\section{To cite this version:}

Benoît Lebon, Jorge Peixinho, Shun Ishizaka, Yuji Tasaka. Subcritical transition to turbulence in a sudden circular pipe expansion. Journal of Fluid Mechanics, 2018, 849, pp.340-354. 10.1017/jfm.2018.421 . hal-02143286

\section{HAL Id: hal-02143286 \\ https://hal.science/hal-02143286}

Submitted on 29 May 2019

HAL is a multi-disciplinary open access archive for the deposit and dissemination of scientific research documents, whether they are published or not. The documents may come from teaching and research institutions in France or abroad, or from public or private research centers.
L'archive ouverte pluridisciplinaire HAL, est destinée au dépôt et à la diffusion de documents scientifiques de niveau recherche, publiés ou non, émanant des établissements d'enseignement et de recherche français ou étrangers, des laboratoires publics ou privés. 


\title{
Subcritical transition to turbulence in a sudden circular pipe expansion
}

\author{
Benoît Lebon $^{1}$, Jorge Peixinho ${ }^{1} \dagger$, Shun Ishizaka ${ }^{2}$ and Yuji Tasaka ${ }^{2}$ \\ ${ }^{1}$ Laboratoire Ondes et Milieux Complexes, CNRS and Université Le Havre Normandie, \\ 76600 Le Havre, France \\ ${ }^{2}$ Laboratory for Flow Control, Hokkaido University, 060-8628 Sapporo, Japan
}

(Received $\mathrm{xx}$; revised $\mathrm{xx}$; accepted $\mathrm{xx}$ )

The results of experiments on the flow through a circular sudden expansion pipe at moderate Reynolds numbers are presented. At five diameters upstream of the expansion, laminar flow was disturbed by a (constant) crossflow jet, a suction or a (periodic in-out) synthetic jet from a hole on the wall. When the disturbance exceeded a critical value of the control parameter depending on the Reynolds numbers, localised turbulent patches formed downstream the expansion at fixed axial positions. For the crossflow jet, the onset of turbulent patches is related to the velocity ratio of the mean jet velocity to the mean pipe velocity. At low velocity ratio, turbulent patches formed intermittently. For the suction disturbance, the flow experienced a strong asymmetry of the recirculation region and required a larger velocity ratio before the turbulent patch formed. For the synthetic jet, the amplification of wavy disturbances into turbulent patches and their axial positions are controlled by the driving frequency. Overall, these results suggest the existence of different mechanisms for the development of localised turbulent patches.

Key words: Transition to turbulence, jets

\section{Introduction}

The flow of a viscous fluid in a cylindrical expansion pipe has been studied for practical reasons in mechanical engineering (Rennels \& Hudson 2012), food processing (Alberini et al. 2015) and also in biomedical applications (Vétel et al. 2008). Although major researches of pipe flow had been conducted in straight pipe, many practical pipe facilities are not straight but have bends, expansions, contractions and so on. Moreover, the flow in a circular pipe with an axisymmetric sudden expansion, at flow rates around the transition to turbulence, is a test case in physiological problems (Zmijanovic et al. 2017). This flow consists of a decelerating round jet issuing from the inlet pipe. In addition, the corners of the expansion are responsible for the formation of a recirculation region. The present investigation deals with the case of a laminar flow at the inlet perturbed by a finite amplitude disturbance, which travels and eventually relaminarises downstream of the expansion, if the Reynolds number is below one thousand. The Reynolds number here is based on the inlet diameter $d$ as $R e=U d / \nu$ where, $U$ is the inlet mean flow velocity and $\nu$ is the kinematic viscosity.

The transition to unsteady flows in sudden pipe expansions have been investigated experimentally (Sreenivasan \& Strykowski 1983; Pak et al. 1990; Hammad et al. 1999;

$\dagger$ Email address for correspondence: jorge.peixinho@univ-lehavre.fr 
Mullin et al. 2009) and numerically (Sanmiguel-Rojas et al. 2010; Cantwell et al. 2010; Sanmiguel-Rojas \& Mullin 2012; Selvam et al. 2016). In all these investigations, flow separation after the expansion and reattachment downstream led to the formation of an elongated recirculation region near the wall, which axial extent grows linearly with the Reynolds number. Cantwell et al. (2010) showed numerically that infinitesimal perturbations can grow for $R e>26$ and experience significant transient energy growth as $R e$ increases. The maximum optimal energy growth values of the first azimuthal mode are of the order of $10^{3}$ and $10^{6}$ for $R e=600$ and 1200, respectively. Experimentally, Mullin et al. (2009) observed the flow becomes asymmetric from $R e=1139 \pm 10$ and the resulting steady flow becomes time dependent at a critical Reynolds number of $R e=$ $1453 \pm 41$. These values are much smaller than the predictions of recent numerical stability calculations: $R e=3273$ by Sanmiguel-Rojas et al. (2010) or $R e=5080 \pm 5$ by Cliffe et al. (2012). Both works have used extended computational domain of several hundreds of diameters. This disagreement between numerical simulations and experiments rises the question of the subcritical behaviour of the flow to finite amplitude disturbances. Several numerical studies (Sanmiguel-Rojas \& Mullin 2012; Selvam et al. 2016) have found early transition due to finite amplitude perturbations using a tilt type perturbation (along one radial direction) or a vortex type perturbation (along the axial direction with a diameter of a quarter of the inlet pipe diameter), which lead to three-dimensional instabilities and localised turbulent patches. These different perturbations also have different thresholds for transition. Note that in the subcritical regime, the localised turbulent patch relaminarises after the disturbance is removed.

When trying to make connections between theoretical and numerical simulations and experimental observations, a precise temporal and spatial specification of a mathematical perturbation is difficult to replicate in experiments. Ideally, a Dirac $\delta$ function disturbance is required all around the circumference of the pipe to maintain azimuthal symmetry with zero-net-mass-flux added to the main flow. Some disturbance systems have achieved zero injection and/or subtraction of fluid through slits or holes (Draad et al. 1998; Han et al. 2000). Yet, these disturbance systems created spatially localised turbulent puffs. Practically, the most common disturbance is a single-jet through a relatively small hole on the pipe wall (Darbyshire \& Mullin 1995; Hof et al. 2003; Peixinho \& Mullin 2007; Tasaka et al. 2010; Philip \& Cohen 2010; Mehta \& Cohen 2016). The main effect of the crossflow jet is to break the symmetry in a direction perpendicular to the axis. Cross-sectional views of pipe flow downstream the crossflow jet have shown the growth of streaks and hairpins vortices (Mellibovsky \& Meseguer 2007; Philip \& Cohen 2010; Åsén et al. 2010).

This paper has the aim to quantify the effect of finite amplitude disturbances, specifically crossflow, suction and zero-net-mass-flux synthetic (periodic) jet disturbance on expansion pipe flow. Although synthetic jets have been used to control turbulent flow separation and boundary layer flow structures leading to hairpin vortices or stretched vortex rings (Jabbal \& Zhong 2008; Tang et al. 2014), there is a need for quantitative data in the case of transition to localised turbulence in sudden expansion pipe flow.

A second aim is more general. Using different types of disturbances and quantifying the thresholds for transition to localised turbulence depending on the various parameters, including $R e$, it is believed the resonator, which is the ability of the flow to self-sustain perturbations, or amplifier dynamics of the sudden expansion pipe flow can be clarified. In $\S 2$, the experimental setup, the disturbances mechanisms and the visualisation method are presented. In $\S 3$, the results and discussion of the flow visualisation and the thresholds of the different disturbance mechanisms for turbulent patches are presented. Finally, some conclusions are drawn in $\S 4$. 
(a)

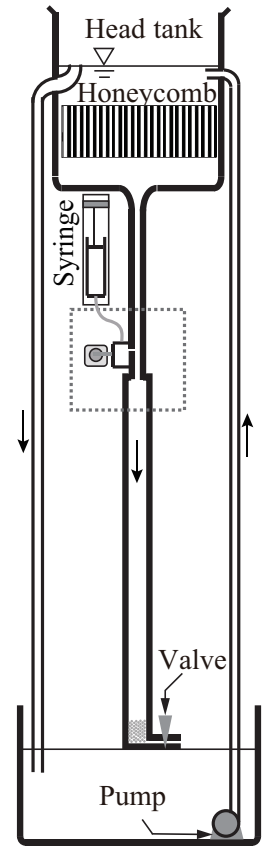

(b)
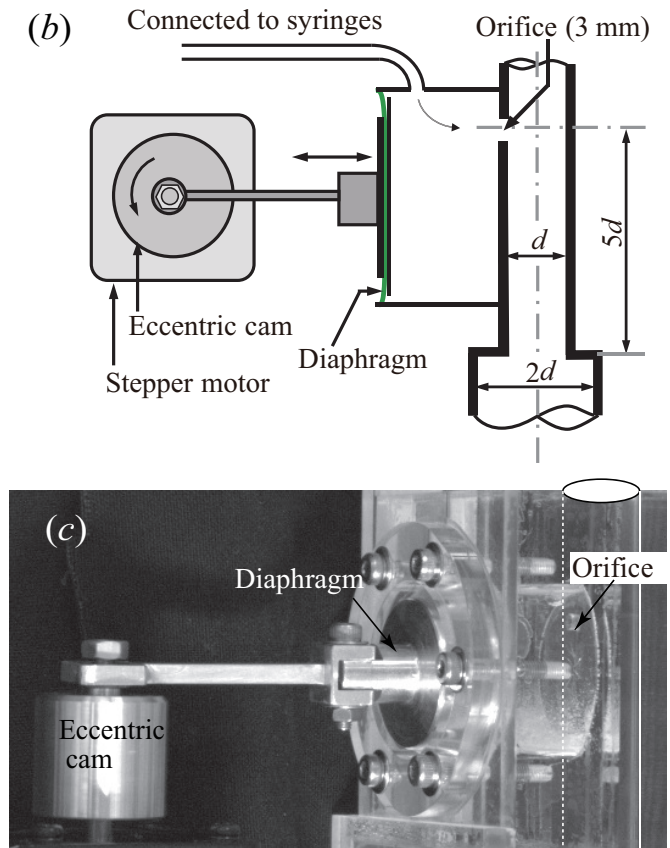

Figure 1. (a) Schematic diagram of the sudden expansion pipe flow apparatus. (b) Schematic of the disturbance mechanism, where the syringe pump connection is used only for injection or suction. (c) Photograph of the synthetic jet mechanism indicating the eccentric cam, the connecting rod, the silicon membrane labelled diaphragm, the cavity and the orifice to the inlet pipe

\section{Experimental set-up}

A schematic of the apparatus is shown in figure 1( $a)$. The pipe is made of an assembly of $15 \mathrm{~cm}$ long transparent push-fitted plastic tubes. The inlet pipe has a diameter of $d=10 \pm 0.01 \mathrm{~mm}$ and is $52.5 d$ long with a trumpet shape at the inlet to ensure the flow to be fully developed. The following section is $97.5 d$ long with a diameter of $2 d$. The flow is pressure driven. To control the flow rate, the water level into a 50 liters head tank is maintained constant by a spillway and the constant supply of water from a pump. An honeycomb mesh is placed inside the tank to damp unwanted disturbances. The flow rate is then controlled by two fine adjustment valves from Swagelok whose aperture is managed by a graduated screw. The long term temperature stability of the laboratory was controlled to $\pm 1^{\circ} \mathrm{C}$ at a mean temperature of $20^{\circ} \mathrm{C}$. By these means, Reynolds number up to 2000 of better than $1 \%$ was achieved.

Figure $1(b)$ is a schematic diagram of the disturbance mechanism, which consists of a hole of $3 \mathrm{~mm}$ diameter (labeled orifice) located at $5 d$ upstream the expansion. In injection mode, a crossflow jet was generated using a $N E-1600$ programmable syringe pump with glass syringes. The disturbance generator is a variant of the system developed by Hof et al. (2003) and, as in their case, the duration and amplitude of the perturbation can be changed independently. They showed that long duration disturbances, corresponding to the localised turbulence length, lead to a well defined threshold for sustained turbulence. Here the duration of the disturbance is also kept long, not less than $90 \mathrm{~s}$. The main parameter describing the amplitude of the disturbance is $V_{R}=\left|V_{j}\right| / U$, where $V_{j}$ is the jet mean velocity. The same system can be used in suction mode to disturb the flow. Suction has been used in the past as a promising method for maintaining laminar 
boundary-layer flows (Pfenninger 1961). Here the suction is through a single hole and the amplitude is also quantified using $V_{R}$.

For the synthetic jet, no syringe pump is used, instead, a motor drives the oscillation of a diaphragm into a cylindrical cavity of $3 \mathrm{~cm}$ in diameter and $2 \mathrm{~cm}$ in height, connected to the inlet pipe through the $3 \mathrm{~mm}$ hole. A photography of the synthetic jet disturbance system is shown in figure $1(c)$. Two cams with an eccentricity $A=50$ and $100 \mu \mathrm{m}$ have been used leading to different oscillation amplitudes. The frequency is set by the rotation speed of the stepper motor, Orientalmotor PKP series. The motor is computer controlled through an interface card Arduino UNO. The step resolution, which is $0.9^{\circ}$, allows oscillations from 0.01 to $10 \mathrm{~Hz}$. The precision of the rotation speed is below $1 \%$ for frequencies under $4 \mathrm{~Hz}$ and up to $5 \%$ at $10 \mathrm{~Hz}$.

In order to validate the experiment, the axial length, $L$, of the recirculation region as a function of $R e$ is shown in figure 2. The photographs in figures $2(a),(b)$ and $(c)$ show the particles path-lines and the extent of the recirculation region for $R e=9,60$ and 107 using long exposure type photography. The flow is seeded with $50 \mu \mathrm{m}$ spherical particles made of polyamide, with a density of $1.018 \mathrm{~g} / \mathrm{cm}^{3}$. The particles are lightened by a $1.5 \mathrm{~W}$ green laser sheet. A lens of focal $300 \mathrm{~mm}$ is used to zoom at the expansion area. The distortion of the image caused by the pipe's curvature is reduced by a visualisation box filled with water. Images of the resulting particles field are recorded by a camera which has a sensor of $1280 \times 800$ pixels, $20 \mu \mathrm{m}$ width, leading to a resolution of $69 \mu \mathrm{m} / \mathrm{px}$. The images were acquired at $15 \mathrm{~Hz}$ for $R e=9$ and at $20 \mathrm{~Hz}$ for $R e=60$ and 107, with an exposure time of $1 \mathrm{~ms}$. The images are then combined to reveal the path-lines of particles. In addition, the recirculation region was monitored using two-dimensional particle image velocimetry (PIV). The acquisitions were performed with a high resolution camera $(2560 \times 2060$ pixels $)$ combined with a zoom (Sigma) of focal 28-300 mm allowing a resolution of $50 \mu \mathrm{m} / \mathrm{px}$. The flow was seeded with 8-11 $\mu$ m hollow-glass spheres with a density of $1.1 \mathrm{~g} / \mathrm{ml}$ and lightened by a laser (DualPower 135-15). The vectors fields are computed from Dantec Dynamic Studio software in order to extract $L$. As $R e$ increases, the axial recirculation length also increases. In a circular pipe, the recirculation is axisymmetric and pointed in the downstream direction. The PIV experiments show, in figure $2(d)$, that $L$, is of the form $L / d=0.044 R e$, which agrees well with previous experimental (Back \& Roschke 1972; Iribarne et al. 1972; Latornell \& Pollard 1986; Hammad et al. 1999) and numerical (Cantwell et al. 2010; Selvam et al. 2015) studies for sudden expansion flow. The position of the reattachment point in the downstream part of the recirculation is not trivial to locate accurately because of the wall curvature and light distortion effects. Hence the onset of asymmetric growth could not be detected with the current resolution and is limited to a relatively small range of $R e$.

The flow state was monitored using Kalliroscopes as the flow visualant. In addition, some experiments were conducted using fluorescein dye. To illuminate the flow, a thin vertical sheet of light was formed using a series of white light sources arranged along the length of the pipe. In some cases a laser sheet was preferred. A camera, mounted on a tripod support, was used to record and monitor the evolution of disturbances. Typical photographs of the disturbed flow are given in the top image of figure $6(a),(b)$ and $(c)$.

\section{Results and discussion}

The results are presented in the form of finite amplitude threshold curves for subcritical transition and space-time diagrams. In injection mode, the threshold for localised turbulence lies in the range $0.15 \leqslant V_{R} \leqslant 1.3$ for $420 \leqslant R e \leqslant 1100$ and hairpin vortex structure has been revealed, whereas in suction mode, the threshold is higher and a 

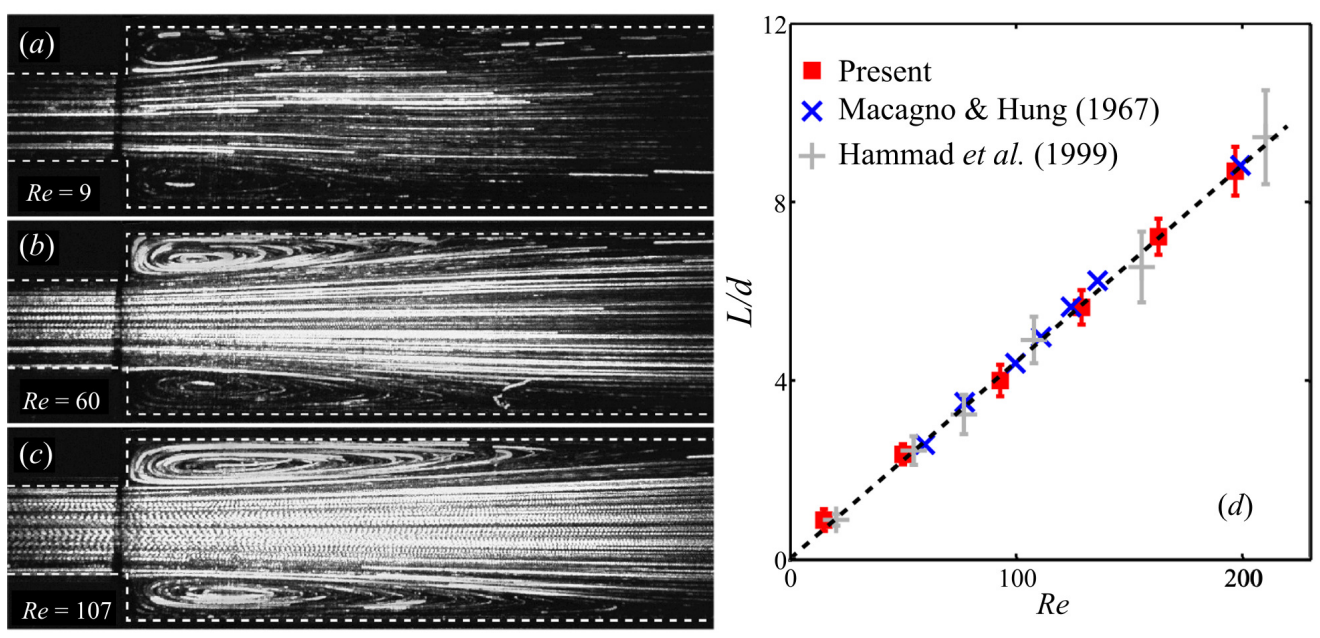

Figure 2. Particle path-lines of the flow downstream the expansion for $(a) R e=9,(b) R e=60$ and $(c) R e=107$. The dashed lines indicate the contour of the expansion. (d) Recirculation length, $L / d$, versus $R e . \times$ and + correspond to previous experimental results (Macagno \& Hung 1967; Hammad et al. 1999). The dashed line represents the best-fit proportionality $L / d=0.044 R e$.

significant asymmetry of the flow is observed before the onset of localised turbulence. The final set of results is concerned with synthetic jet disturbances through the same hole. The dependence of the frequency on the amplification of the disturbance along the expansion pipe is quantified.

\subsection{Single-jet disturbance}

In this experimental set-up at $R e \gtrsim 1200$, disordered flow is initiated naturally by imperfections, roughness, fluid impurities, etc. For $400 \lesssim R e \lesssim 1200$, the flow remains laminar and only disturbances of sufficiently large amplitude can initiate localised turbulence. For uniform pipe flow disturbed by crossflow jets, the dependency of the flux, the duration, the orientation and the jet diameter was already investigated (Hof et al. 2003; Peixinho \& Mullin 2007). Specifically, the critical flux for turbulence is independent of the injection time when the dimensionless disturbed length is larger than 6 pipe diameters. It is also known that smaller jet diameters lead to lower flux thresholds (Peixinho \& Mullin 2007; Tasaka et al. 2010).

The effect of crossflow jet to the sudden expansion pipe flow is shown in figure 3 . At small $V_{R}$, the jet modifies the incoming velocity profile, but the flow recovers its laminar state downstream while the disturbance in injecting. The flow is considered transient when the laminar state is disturbed for a small fraction of the injection duration (e.g. see figure $4(a))$ and it is considered above the threshold for localised turbulence when the flow is disturbed for most of the duration of the applied disturbance (e.g. see figure $4(b))$. In figure 3 , the critical velocity ratio, $V_{R}$, decreases with increasing $R e$ indicating that the pipe is more and more sensitive to the jet disturbance. A least-squares fit to the single-jet data gives a good description through a power law: $V_{R} \propto R e^{-2.3 \pm 0.1}$. The scaling for subcritical turbulence is fairly abrupt and could be related to the amplification behaviour of the expansion flow. Clearly, the range of $R e$ is limited. Furthermore, the scaling can not hold for smaller $R e$. For $R e \lesssim 400$, disturbances can not be sustained downstream. 


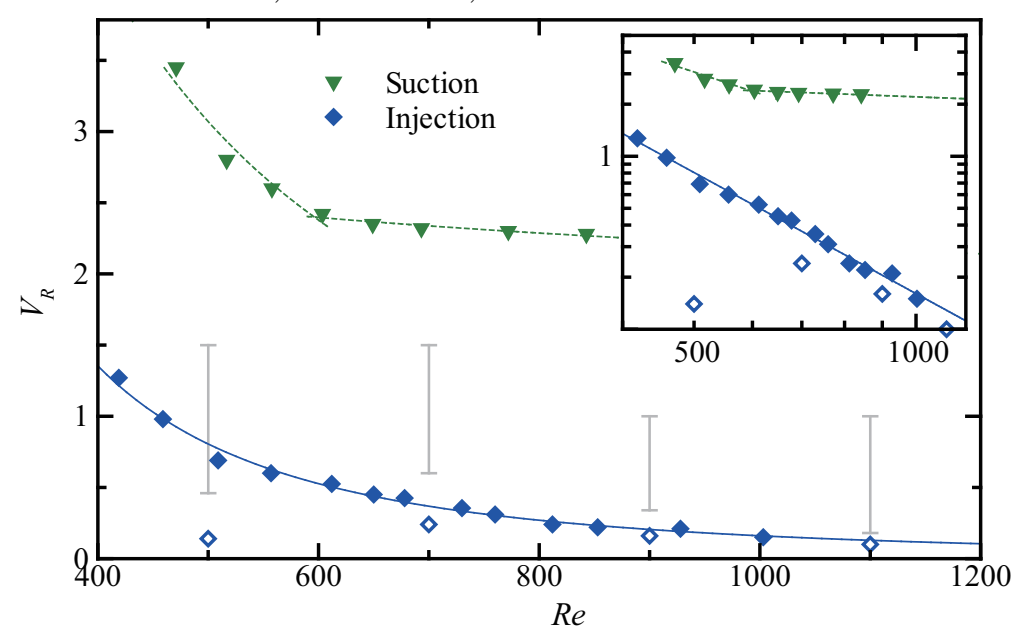

FIGURE 3. Critical velocity ratio, $V_{R}$, for the onset of localised turbulent patch for single-jet and suction as a function of $R e$. The continuous line is a power law fit: $R e^{-2.3}$ and the dashed line is a guide to the eye. The error bars indicate the range of parameters where hairpin vortices are observed and open symbols represent visual laminar transient disturbances for the single-jet. The inset is the same results in log-log scale.

Once $V_{R}$ exceeds the threshold, localised turbulence is formed downstream. Typical examples of transient, intermittent and localised turbulent patch are show in the top image of figure $6(a),(b)$ and $(c)$, respectively. Figure 4 shows examples of space-time diagrams of the brightness along the centreline axis of the pipe for $R e=700$ and 900. The vertical black lines mark the junction between pipe sections. As the singlejet is started at $t=0$, the disturbance moves downstream and develops before the localised turbulent patch forms downstream the expansion at $x / d \simeq 3$. The oblique lines on the diagram represent particles that move downstream and the slope can be related to the mean advection speed of these particles. At $t / t_{i n j}=1, t_{i n j}$ being the injection total duration, the injection is stopped and the turbulent localised patch downstream the expansion decays as it is carried downstream. Below the threshold, $V_{R}$ is sufficient to produce disturbed flow that evolves as transient localised turbulence. It is supposed that the injection induces a counter rotating vortex, typical of crossflow jet (Camussi et al. 2002; Vétel et al. 2008; Ilak et al. 2012; Karagozian 2014), which breaks the azimuthal symmetry of the recirculation region downstream the expansion and induces non-axisymmetric modes as proposed by Cantwell et al. (2010). Yet, the quantitative identification of such azimuthal modes from the experiments would require three-dimensional PIV and is out of scope of the present study. An example of transient localised turbulence is presented in figure $4(a)$ for $R e=700$ and $V_{R}=0.32$. The movie 1 is a example of localised turbulent for $R e=700$ and $V_{R}=0.35$.

The dependence of the dynamics of disturbances on various $V_{R}$ and $R e$ were studied through space-time diagrams. These diagrams can be characterised using $t_{c}$, the onset time for the turbulent patch and the patch duration, $t_{\text {patch }}$, as sketched by the arrows in figure $4(b)$. The results of a systematic investigation are presented in figure 5 and show a decrease of $t_{c}$ and a rapid increase and saturation of $t_{\text {patch }}$ as $V_{R}$ increases. When scaled by the advection time, $U / d$, the data for different $R e$ and different $V_{R}$ seem to collapse. All together, the data suggest that the subcritical behaviour for single-jets depends only on $V_{R}$, the intensity of the jet compared to the mean pipe velocity.

For low $V_{R}$, transient growth and decay of the turbulent patches are observed. In order 

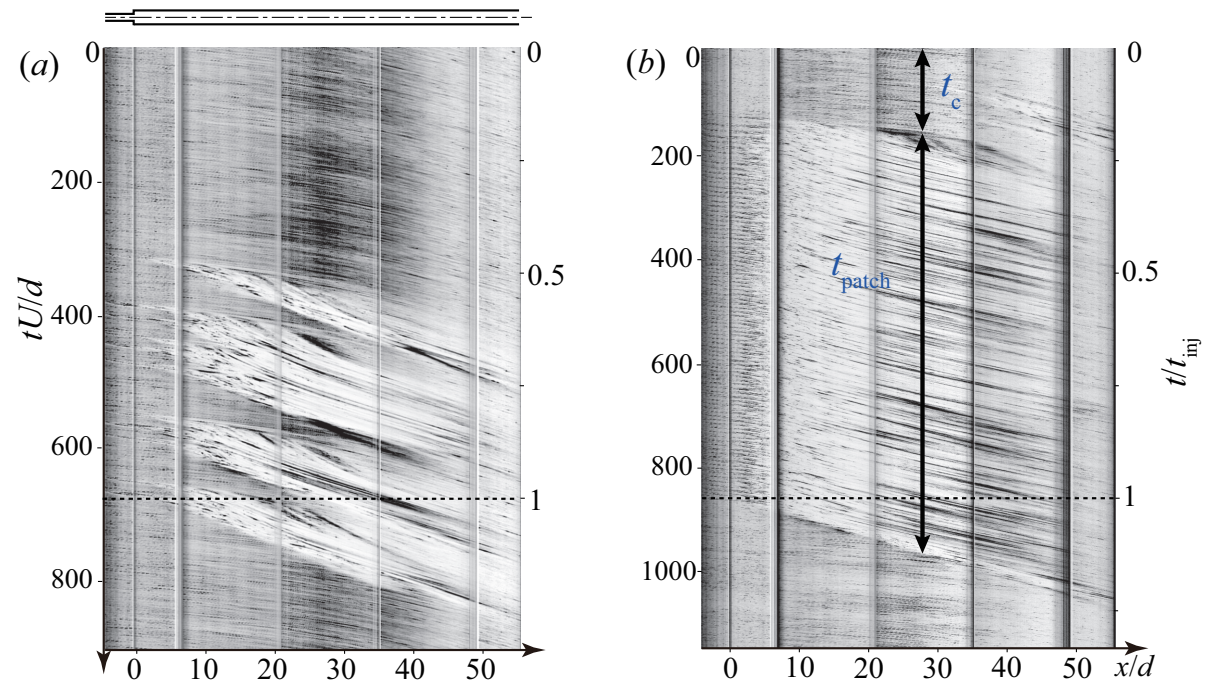

Figure 4. Space-time diagram of localised turbulence patches $(a)$ below threshold (transient) at $R e=700$ and $V_{R}=0.32,(b)$ above threshold at $R e=900$ and $V_{R}=0.54$
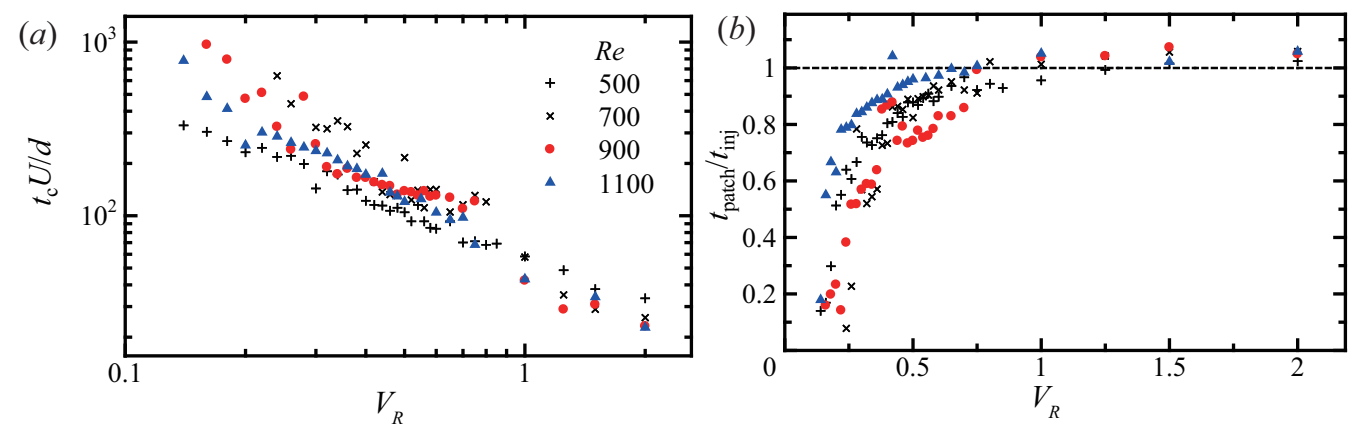

Figure 5. (a) Onset time, $t_{c}$, of the turbulent localised patch as a function of $R e$ and $(b)$ variations of duration time of a turbulent patch, $t_{\mathrm{patch}}$, with respect to $V_{R}$ at different $R e$. The dotted line indicates the end of the injection.

to describe the spatial-temporal dynamics, figure 6 shows spatio-temporal diagrams made at different axial locations along the diameter for $R e=700$. In figure $6(a), V_{R}=0.26$, the disturbance breaks first at $20 d$ from the expansion, grows slightly up to $x / d=30$ and decays. In figure $6(b)$, for $V_{R}=0.34$, an intermittent regime is observed. Finally, in figure $6(c)$ for $V_{R}=0.75$, the growth remains localised between $x / d=10$ and 40 .

\subsection{Hairpin vortices}

Hairpins vortices has been observed before in uniform pipes (Peixinho \& Mullin 2007; Tasaka et al. 2010; Åsén et al. 2010) or expansion pipe (Vétel et al. 2008). Here these have been observed specifically for the single-jet disturbance and their domain of existence is indicated by error bars in figure 3 above the threshold for localised turbulent patches. It is interesting to note that the domain of existence for hairpin vortices appears at $0.5 \lesssim V_{R} \lesssim 1.5$. This range of $V_{R}$ is in agreement with the range reported in previous studies of jet in crossflow in large or unbounded domains (Camussi et al. 2002; Ilak et al. 2012; Karagozian 2014). This suggests that counter-rotating vortices pairs are present in the experiments and may induce instabilities. 

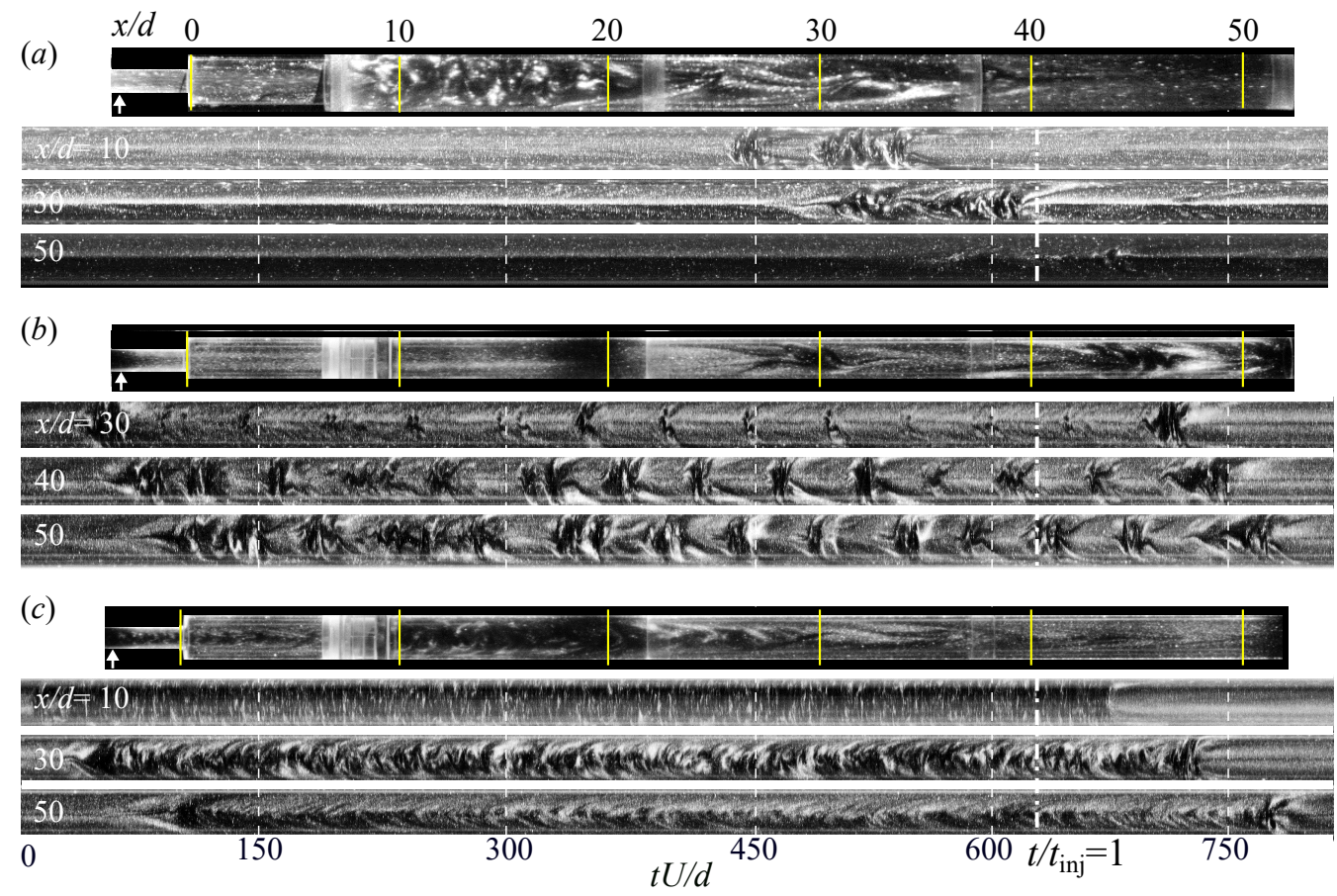

FIGURE 6. Flow visualisation photographs and space-time diagrams of transient growth of disturbances at $R e=700$ for $(a) V_{R}=0.26,(b) 0.34$ and $(c) 0.75$. The arrow on the photographs indicate the position of the single-jet disturbance. The dashed-dot lines on the diagrams indicate the end of the jet injection.
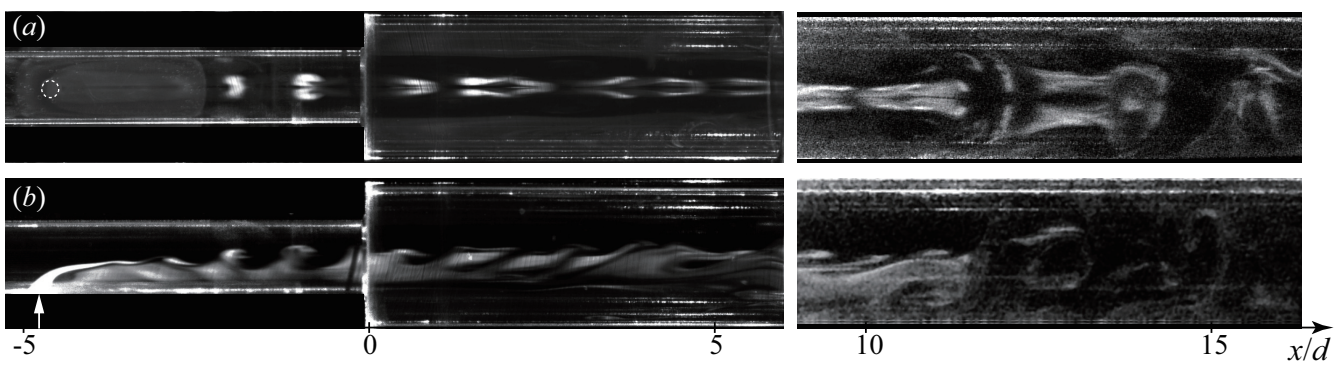

FiguRe 7. Photographs of a development of hairpin vortex array visualised through fluorescein dye taken for $R e=700$ and $V_{R}=0.7 ;(a)$ top view and $(b)$ side view

Figure 7 shows these hairpin vortices emerging from a single-jet disturbance at the inlet, propagating through the expansion and breaking down into a turbulent patch. On side view images in figure $7(b)$, the inclination of the hairpin vortices is enhanced due to the stretching effect when passing through the expansion.

\subsection{Suction disturbance}

In the case of the suction disturbance, the effect of the aspiration is to distort the inlet velocity profile that induces a strong asymmetry of the flow. A snapshot of the base flow without disturbance is shown in figure $8(a)$, and the distorted flows for different $V_{R}$ in figures $8(b)$ and $8(d)$, respectively below and above the critical value of $V_{R}$. The vertical white bands correspond to the connections between the pipe sections and the black 


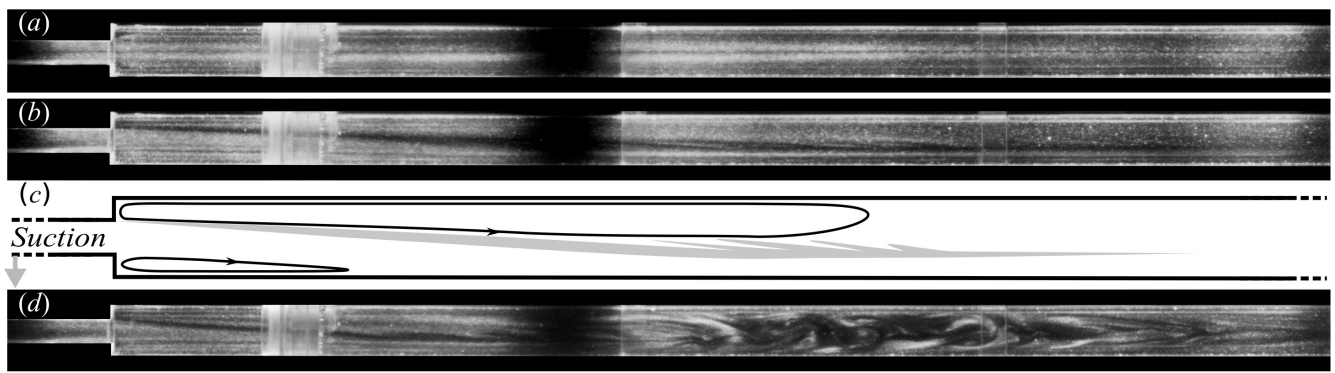

Figure 8. Photographs for the suction disturbance for $R e=600$. (a) Laminar flow: $V_{R}=0$, (b) low amplitude suction at $V_{R}=2.2,(c)$ schematic of the streamlines of case $(b)$, where the grey area represents the shear zone, and $(d)$ suction at $V_{R}=2.5$

shadow corresponds to the separation between the two lamps used during acquisition. Figure $8(c)$ is a sketch illustrating the flow deformation and the position of shear-unstable regions in gray. Specifically, the recirculation area on the opposite side of the aspiration gets several times bigger.

Below the critical value of $V_{R}$, transient wavy disturbances appear at the boundary between the main flow and the recirculation area. These are relaminarised without initiating localised turbulence. The movie 2 provided in the supplementary material corresponds to the case $R e=600$ and $V_{R}=2.2$. On the contrary, in figure $8(d)$, the $V_{R}$ is above the critical value. The amplification of instability between the main flow and the recirculation area is observed, the previously mentioned wavy disturbances grow and lead to the formation of a turbulent patch fixed in space. Small disturbances are formed and amplified during the aspiration, sustaining the turbulent patch. In addition, a region of high shear appears at $x / d \approx 15$ from the expansion and the analysis of the brightness intensity on the centreline indicates the existence of small amplitude oscillations with a frequency of about $0.43 \mathrm{~Hz}$. Such oscillations and frequency have been reported before in numerical simulations (Boujo et al. 2013; Selvam et al. 2015) and their authors suggested Kelvin-Helmholtz instabilities between the main flow and the recirculation region behind an expansion or a bump.

The finite amplitude thresholds of the suction disturbance for the onset of localised turbulent patch are also presented in figure 3. Distinct scalings are observed for the jet and the suction. It is also clear that larger $V_{R}$ are required to cause transition. Indeed, the suction preserves the recirculation region and somehow selects axisymmetric modes that require higher threshold to induce instability. This is consistent with the numerical study of Cantwell et al. (2010) which showed that the energy growth of azimuthal modes is larger than axisymmetric ones.

Note that the injection and suction modify the flux in the inlet pipe. Moreover, the scaling for the critical $V_{R}$ has a different behaviour as the suction seems to reach a plateau corresponding to a shear consistent with the constant critical velocity required to cause Kelvin-Helmholtz instability. When the suction is stopped, the flow recovers the axisymmetric recirculation pattern after a short transient time.

\subsection{Synthetic jet disturbance}

In the previous subsections, crossflow jet and suction have been tested in a systematic way and indicate clear thresholds for localised turbulence. Now, these disturbances are combined to achieve a periodic zero-net-mass-flux disturbance, since an equal quantity of fluid was pushed then pulled in and out of the pipe through the $3 \mathrm{~mm}$ diameter hole. Although periodic excitation is very different from constant-flux disturbances, the 

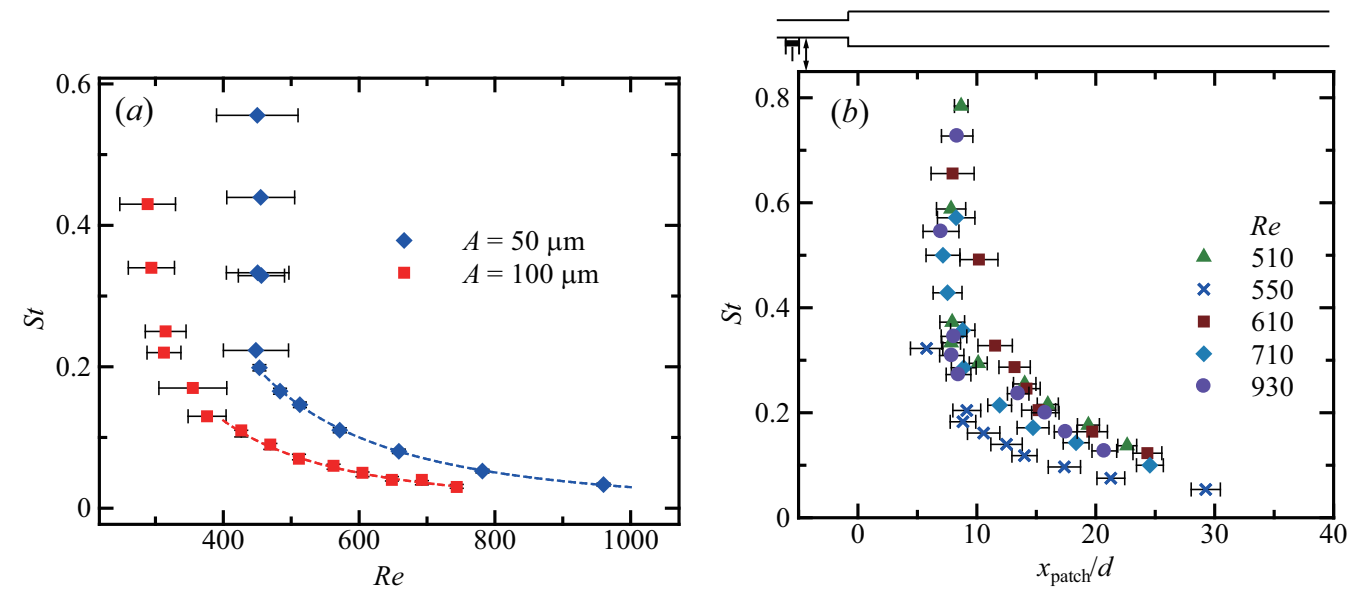

Figure 9. Stability of the synthetic jet disturbance: $(a) S t$ versus $R e$. The lines are last-square fits: $S t \propto R e^{-2.2 \pm 0.2}$. (b) $S t$ versus turbulent patch position, $x_{\text {patch }} / d$, for different $R e$ and $A=50 \mu \mathrm{m}$. The schematic diagram indicates the position of the disturbance, the expansion and the turbulent patch.

periodic injection and suction should lead to essentially the same effect at very low frequency of oscillation.

Hence, the key parameter is the frequency of the oscillations, $f$, which is made dimensionless through a Strouhal number: $S t=f d / U$. The synthetic disturbance was systematically tested for different $R e$ and $S t$ values. Figure $9(a)$ shows the threshold for localised turbulence as a function of $R e$ for two initial oscillation amplitudes: $A=50$ and $100 \mu \mathrm{m}$. Note that the values of $A$ are relatively small in this study in order to agree to transient growth theory. The general shape of the threshold data is a sharp cutoff at low $R e$ and a decreasing curve as $R e$ increases, indicating the expansion pipe flow is more sensitive to disturbances of low frequency as $R e$ increases. The threshold away from the cutoff can be fitted with a power law: $R e^{-2.4 \pm 0.2}$ with an exponent similar to the exponent for the single-jet. This is because the maximum $V_{R}$ can transiently reach values typical of the threshold for single-jet. A systematic shift is observed toward low $R e$ and low $S t$ as the eccentricity of the cam, $A$, is increased. For high $S t$, the behaviour of the synthetic jet is basically the periodic formation of stretched vortex rings. As there is a sharp cutoff, the method to estimate the threshold is to keep the oscillation frequency $f$ constant and vary Re. This leads to horizontal error bars with the left-hand error bar indicating rapid decay of the disturbance and the right-hand a persistence of the disordered motion.

The disturbance involves and forms a localised turbulent patch, which is about $25 d$ long with an active core and a decaying waves downstream. The location of the active core, $x_{\text {patch }}$, remains at a steady axial position from the expansion as long as the disturbance is applied. $x_{\text {patch }}$ varies sensitively upon $S t$ and is almost independent of $R e$ as shown in figure $9(b)$. High $S t$ disturbances caused disturbed flow at $x \simeq 7 d$. At $S t \approx 0.1$, oscillatory disturbances induced unsteady flow further downstream at $x \simeq 25 d$ from the expansion.

Transient growth of infinitesimal perturbations has been discussed in various flows (Westin et al. 1998; Marais et al. 2011; Boujo et al. 2013; Klotz \& Wesfreid 2017) where the position of the centroid of perturbations is reported to move upstream depending on $R e$ and the perturbation frequency. For expansion pipe, the transient growth analysis from Cantwell et al. (2010) indicates that stochastic noise-driven flow at $R e=600$ and 900 have non-axisymmetric azimuthal modes of disturbances with a centroid of 
perturbation energy at $x / d=18$ and 32 , respectively. This very close to the measured $x_{\text {patch }}$ reported in figure $9(b)$. However, the experiment did not show clear periodic optimal flow structures. The simulations of Sanmiguel-Rojas et al. (2010) have predicted the first unstable mode at $x \approx 150 d$ from the expansion for $R e \simeq 3273$ and $S t \simeq 0.13$. It suggests that smaller amplitude disturbance could initiate disturbed flow further downstream. Anyway, in the present experiment, when the disturbance is stopped, the patch is no longer sustained.

In figure 10, a photograph and space-time diagrams along the pipe diameter at fixed axial positions, where the turbulent patch starts to develop, are presented. Using fluorescein solutions, with a mass concentration of $0.001 \mathrm{mg} / \mathrm{l}$, injected at the inlet of the pipe with a flow-rate of $1 \mathrm{ml} / \mathrm{min}$, the growth of the wavy disturbance can be monitored. The dye travels downstream, passes in front of the disturbance mechanism and through the expansion. Downstream the expansion, the periodic patterns are captured using the cameras.

Space-time diagrams along the diameter are produced, which show the amplification of the oscillations. Figure $10(b),(c)$ and $(d)$ present space-time diagrams for $S t=0.1$, 0.17 and 0.42 , respectively. Note the $x$-position has to be adjusted and the maximum amplification growth changes with $S t$. For $S t=0.1$, the amplification occurs at $x \simeq 26$ and the wavy disturbance is merely disturbed. For $S t=0.17$, the maximum amplification occurs at $x / d \simeq 13$ and the space-time pattern contains the main wave and presumably one harmonic. For $S t=0.42$, the maximum amplification occurs at $x / d \simeq 5$ and seems to contain several harmonics. Yet, no frequency shift is observed upon the main frequency according to fast Fourier transform analysis of the light intensity (not shown). Hence, the synthetic jet disturbance in the range of parameter studied behaves as an amplifier. Further, the space-time diagrams suggest the instabilities are convective as the flow contains weak or almost no swirl around the axes (Miranda-Barea et al. 2016).

The results seem consistent with previous experiments from Chun \& Sung (1996), where the turbulent separation flow downstream a backward-facing step was controlled using a synthetic jet from a thin slit at the corner. The corner location has also been shown to be highly sensitive to disturbances in expansion pipe flow (Sreenivasan \& Strykowski 1983; Cantwell et al. 2010). It is anticipated that disturbances introduced further upstream from the expansion may need to be compensated with larger amplitudes to observe transition. Yet, the scalings exponents for the thresholds could still hold and may be used to control the drag or the mixing in the expansion.

\section{Conclusions}

The results of the stability of the flow past a sudden axisymmetric expansion experiencing disturbances have been described. Upstream and downstream pipe sections made of clear transparent material were used to make these observations. Different disturbance mechanisms (i) a single crossflow jet, (ii) a suction and (iii) a synthetic jet have been tested. With increasing values of the Reynolds number, based on the upstream flow conditions, the flow remains steady and the asymmetry becomes more pronounced for $R e \gtrsim R e_{c}$, where $R e_{c} \approx 1200$ is the natural critical Reynolds number of the apparatus. For $R e \approx 300$, the flow becomes sensitive to disturbances, thus the flow visualisation indicates the presence of localised turbulent patches. The single-jet is more efficient than the suction in the sense it requires less flux to initiate turbulence. The threshold amplitude for the simple injection scales with the Reynolds number with power law exponents of -2.3. This exponent is consistent with the possibility of transient growth in the initial steps of the transition process. Moreover, horseshoe structures have been observed for a 


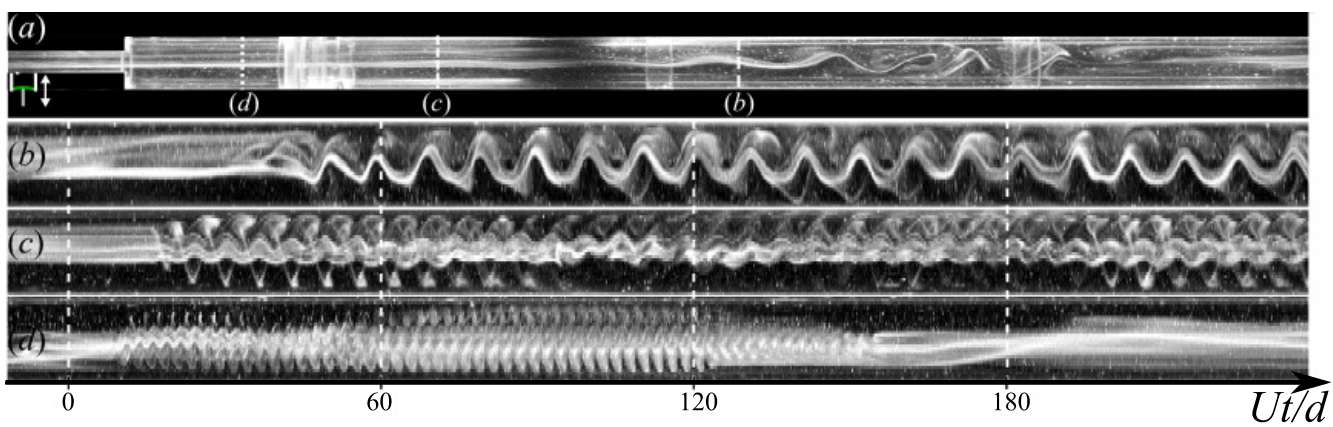

FiguRE 10. Effect of the synthetic jet. (a) A snapshot of the propagating oscillating disturbance from fluoresceine visualisation images of the synthetic jet disturbance at $R e=600$ and $S t=0.1$. A movie is provided in the supplementary material (see movie3.mp4). Space-time diagrams at constant $x / d$ along the diameter for $(b) S t=0.1$ at $x / d \simeq 26,(c) S t=0.17$ at $x / d \simeq 13$ and (d) $S t=0.42$ at $x / d \simeq 5$. The location $x / d$ corresponds to the trailing edge (upstream) of the disturbed flow.

range of velocity ratio and these are known to appear as a result of crossflow instability and play a central role in transition to turbulence. The suction disturbance produces a favourable pressure gradient towards the wall and subsequently a strong asymmetry of the recirculation region downstream the expansion, which nested a shear region between the recirculation and the decelerating jet suggesting a Kelvin-Helmholtz instability.

The stability diagram of the synthetic jet, a zero-net-mass-flux disturbance, has evidenced a threshold at large $R e$ with a similar scaling as the crossflow jet. For low Reynolds number, the synthetic jet has a sharp cutoff for high frequency, which critical Reynolds number is dependent on the oscillation amplitude. At low frequency, the periodic forcing has the advantage to select the axial position where the oscillatory flow is amplified and eventually breaks into a localised turbulent patch. In the future, it is planned to study the effect of the expansion ratio, as plane expansions studies (Lanzerstorfer \& Kuhlmann 2012) have shown that the ratio leads to different transition mechanisms depending on the amplification processes: pure centrifugal or elliptical.

Our work has benefited from many discussions with Tom Mullin. We also thank Bernard Lechelle and Toshiyuki Sampo for their technical and engineering support. The authors acknowledge the financial support of the Agence Nationale de la Recherche (ANR), through the project 'TRANSFLOW' and the programme 'Investissement d'Avenir', LabEx EMC3. Visitation of SI at LOMC was supported by JSPS open partnership collaboration research grant. YT was supported by JSPS KAKENHI $15 \mathrm{KK} 0219$ and $16 \mathrm{H} 04260$.

\section{REFERENCES}

Alberini, F., Simmons, M. J. H., Parker, D. J. \& Koutchma, T. 2015 Validation of hydrodynamic and microbial inactivation models for UV-C treatment of milk in a swirltube 'SurePure Turbulator' ${ }^{\mathrm{TM}}$, J. Food Eng. 162, 63-69.

ÅsÉn, P.-O., Kreiss, G. \& Rempfer, D. 2010 Direct numerical simulations of localized disturbances in pipe poiseuille flow. Comput. Fluids 39 (6), 926-935.

BACk, L. H. \& Roschke, E. J. 1972 Shear-layer flow regimes and wave instabilities and reattachment lengths downstream of an abrupt circular channel expansion. J. Appl. Mech. 39 (3), 677-681.

Boujo, E., Ehrenstein, U. \& Gallaire, F. 2013 Open-loop control of noise amplification in a separated boundary layer flow. Phys. Fluids 25 (12), 124106. 
Camussi, R., Guj, G. \& Stella, A. 2002 Experimental study of a jet in a crossflow at very low Reynolds number. J. Fluid Mech. 454, 113-144.

Cantwell, C. D., Barkley, D. \& Blackburn, H. M. 2010 Transient growth analysis of flow through a sudden expansion in a circular pipe. Phys. Fluids 22 (3), 034101.

Chun, K. B. \& Sung, H. J. 1996 Control of turbulent separation flow over a backward-facing step by local forcing. Exp. Fluids 21, 417-426.

Cliffe, K. A., Hall, E. J. C., Houston, P., Phipps, E. T. \& Salinger, A. G. 2012 Adaptivity and a posteriori error control for bifurcation problems III: Incompressible fluid flow in open systems with $\mathrm{O}(2)$ symmetry. J. Sci. Compt. 52 (1), 153-179.

Darbyshire, A. G. \& Mullin, T. 1995 Transition to turbulence in constant-mass-flux pipe flow. J. Fluid Mech. 289, 83-114.

Draad, A. A., Kuiken, G. D. C. \& Nieuwstadt, F. T. M. 1998 Laminar-turbulent transition in pipe flow for Newtonian and non-Newtonian fluids. J. Fluid Mech. 377, 267-312.

Hammad, K. J., ӦтÜGen, M. V. \& ARIK, E. B. 1999 A PIV study of the laminar axisymmetric sudden expansion flow. Exp. Fluids 26 (3), 266-272.

Han, G., Tumin, A. \& Wygnanski, I. 2000 Laminar-turbulent transition in Poiseuille pipe flow subjected to periodic perturbation emanating from the wall. J. Fluid Mech. 419, $1-27$.

Hof, B., Juel, A. \& Mullin, T. 2003 Scaling of the turbulence transition threshold in a pipe. Phys. Rev. Lett. 91 (24), 244502.

Ilak, M., Schlatter, P., Bagheri, S. \& Henningson, D. S. 2012 Bifurcation and stability analysis of a jet in cross-flow: Onset of global instability at a low velocity ratio. J. Fluid Mech. 696, 94-121.

Iribarne, A., Frantisak, F., Hummel, R. L. \& Smith, J. W. 1972 An experimental study of instabilities and other flow properties of a laminar pipe jet. AIChE J. 18 (4), 689-698.

JABBAL, M. \& Zhong, S. 2008 The near wall effect of synthetic jets in a boundary layer. Int. J. Heat Fluid Flow 29 (1), 119-130.

Karagozian, A. R. 2014 The jet in crossflow. Phys. Fluids 26 (10), 1-47.

KLOtz, L. \& Wesfreid, J. E. 2017 Experiments on transient growth of turbulent spots. J. Fluid Mech. 829, R4.

Lanzerstorfer, D. \& Kuhlmann, H. C. 2012 Global stability of multiple solutions in plan sudden-expansion flow. J. Fluid Mech. 693, 1-27.

Latornell, D. J. \& Pollard, A. 1986 Some observations on the evolution of shear layer instabilities in laminar flow through axisymmetric sudden expansions. Phys. Fluids 29 (9), $2828-2835$.

Macagno, E. O. \& Hung, T.-K. 1967 Computational and experimental study of a captive annular eddy. J. Fluid Mech. 28 (1), 43-64.

Marais, C., Godoy-Diana, R., Barkley, D. \& Wesfreid, J. E. 2011 Convective instability in inhomogeneous media: Impulse response in the subcritical cylinder wake. Phys. Fluids 23 (1), 014104.

Menta, V. \& Cohen, J. 2016 Experimental investigation of a low-amplitude transition in pipe flow. Fluid Dyn. Res. 48 (6), 061428.

Mellibovsky, F. \& Meseguer, A. 2007 Pipe flow transition threshold following localized impulsive perturbations. Phys. Fluids 19 (4), 044102.

Miranda-Barea, A., Fabrellas-García, C., Parras, L. \& del Pino, C. 2016 Spin-down in rotating Hagen-Poiseuille flow: A simple criterion to detect the onset of absolute instabilities. J. Fluid Mech. 793, 316-334.

Mullin, T., Seddon, J. R. T., Mantle, M. D. \& Sederman, A. J. 2009 Bifurcation phenomena in the flow through a sudden expansion in a circular pipe. Phys. Fluids 21, 014110.

PAK, B., Cho, Y. I. \& ChoI, S. U. S. 1990 Separation and reattachment of non-newtonian fluid flows in a sudden expansion pipe. J. Non-Newt. Fluid Mech. 37 (2-3), 175-199.

Peixinho, J. \& Mullin, T. 2007 Finite-amplitude thresholds for transition in pipe flow. J. Fluid Mech. 582, 169-178.

Pfenninger, W. 1961 Boundary layer suction experiments with laminar flow at high Reynolds numbers in the inlet length of a tube by various suction methods. In Boundary layer and flow control (ed. G. V. Lachman), pp. 961-980. Pergamon. 
Philip, J. \& Cohen, J. 2010 Formation and decay of coherent structures in pipe flow. J. Fluid Mech. 655, 258-279.

Rennels, D. C. \& Hudson, H. M. 2012 Pipe flow: A practical and comprehensive guide. John Wiley \& Sons.

Sanmiguel-Rojas, E., Del Pino, C. \& Gutiérrez-Montes, C. 2010 Global mode analysis of a pipe flow through a 1:2 axisymmetric sudden expansion. Phys. Fluids 22 (7), 071702.

Sanmiguel-Rojas, E. \& Mullin, T. 2012 Finite-amplitude solutions in flow through a sudden expansion in a circular pipe. J. Fluid Mech. 691, 201-213.

Selvam, K., Peixinho, J. \& Willis, A. P. 2015 Localised turbulence in a circular pipe flow with gradual expansion. J. Fluid Mech. 771, R2.

Selvam, K., Peixinho, J. \& Willis, A. P. 2016 Flow in a circular expansion pipe flow: Effect of a vortex perturbation on localised turbulence. Fluid Dyn. Res. 48 (6), 061418.

Sreenivasan, K. R. \& Strykowski, P. J. 1983 An instability associated with a sudden expansion in a pipe flow. Phys. Fluids 26 (10), 2766-2768.

Tang, H., Salunkhe, P., Zheng, Y., Du, J. \& Wu, Y. 2014 On the use of synthetic jet actuator arrays for active flow separation control. Exp. Therm. Fluid Sci. 57, 1-10.

Tasaka, Y., Schneider, T. M. \& Mullin, T. 2010 Folded edge of turbulence in a pipe. Phys. Rev. Lett. 105 (17), 174502.

Vétel, J., Garon, A., Pelletier, D. \& Farinas, M.-I. 2008 Asymmetry and transition to turbulence in a smooth axisymmetric constriction. J. Fluid Mech. 607, 351-386.

Westin, K. J. A., Bakchinov, A. A., Kozlov, V. V. \& Alfredsson, P. H. 1998 Experiments on localized disturbances in a flat plate boundary layer. Part 1 . The receptivity and evolution of a localized free stream disturbance. Eur. J. Mech. B/Fluids $17(6), 823-846$.

Zmijanovic, V., Mendez, S., Moureau, V. \& Nicoud, F. 2017 About the numerical robustness of biomedical benchmark cases: Interlaboratory FDA's idealized medical device. Int. J. Numer. Method Biomed. Eng. 33 (1). 